\title{
Context-Aware Fuzzy Databases: An application
}

\author{
Cadenas L., José Tomás ${ }^{1,2}$ Marín Ruiz, Nicolás ${ }^{3}$ Vila Miranda, M. Amparo ${ }^{3}$ \\ ${ }^{1}$ Department of Computation and I.T., Simón Bolívar University, Caracas, Venezuela \\ ${ }^{2}$ CAMYTD, FACYT, University of Carabobo, Valencia, Venezuela \\ ${ }^{3}$ Department of Computer Science and A.I., University of Granada, Granada, Spain
}

\begin{abstract}
The way human beings communicate is contextdependent and it is not easily reproduced on traditional computer systems. We propose combined advances in Context-aware Computing and Fuzzy Databases with the aim of producing more intelligent, flexible and personalized systems. We focus on the interaction of users with a Database System where contextual data could affect the output. We present a development of an application for the Venezuelan Children's Orthopedic Hospital. The data for lower limb joints may be imperfect or fuzzy on database queries. We have developed a web application with an interface for mobile devices with Android operating systems.
\end{abstract}

Keywords: Fuzzy Databases, Context-Aware Applications, Intelligent Systems, Flexible Systems, Personalized Systems.

\section{Introduction}

Traditional database systems answer queries accurately and deterministically. This is known as the exact match query paradigm. Consequently, the DBMS guarantees that the same exact answer will be generated every time the query is issued as long as the data do not change [1]. These database systems suffer from the problem recognized as rigidity.

For years, scientists have studied how to extend DBMS to manage imperfect (imprecise, vague) or uncertain data with Fuzzy Databases [2] or Probabilistic Databases [3], among others. The integration of fuzziness in databases is proposed by [4] because of the imprecision present in data and queries. These approaches allow to represent real world data more faithfully as well as flexible database querying.

On the other hand, the implementation of fuzzy domains in database systems lets us represent semantics of descriptive terms according to user individual perceptions [5], so the system has the capacity of personalizing the language used by each user. Another important feature is that many forms of data imperfection involve the notion of gradualness [6]; that is, the concepts used to describe diverse situations do not correspond to elements of a simple crisp set, but to a fuzzy one.

Also, we as humans consider context all the time in all of our activities, while traditional computer systems do not usually take that into account. In fact, a computer system only takes into account the system designers preset context. As a consequence, a computer system's ability to provide relevant information to users gets re- stricted and they end up having to sift through high volumes of information [7].

The notion of gradualness helps us to address the rigidity problem of the exact query paradigm through flexible query conditions. Thus, the database system can offer query answers with a membership degree, useful in case of queries without answers, or use (combined with contextual data) to filter the query answers (in case of far too many answers).

The medical field is an example where users have to manage a great amount of imperfect data and, additionally the context plays a vital role. In [8] it is stated that managing hospital information is a challenge with unique requirements: documents get lost, instructions are unclear, and data are incomplete. These authors define context in hospital as: location (of worker, device, or artifact), timing of exchange, and person's role (doctor, nurse, laboratory personnel, social worker, among others).

In this paper we present a Context-Aware Fuzzy Database web application with mobile interface in the medical field. To attain this, we make use of the framework presented in [9]. The application implements contextual dimensions and lets the user define context dependencies in a friendly way. Particularly, each user can define her/his semantics of descriptive terms using fuzzy domains, allowing custom input data and getting flexible queries according to user needs. The application was developed for the Venezuelan Children's Orthopedic Hospital, and we focus on the Joint Physical Examination accomplished in the gait Analysis Laboratory.

Our proposal looks at the context as it has been considered in other computational areas to develop contextaware applications, in which query results can be affected according to the current user context. Hence, we personalize the user experience without overwhelming her/him with excessive actions, and adding new user friendly DBMS facilities.

We want to contribute to develop intelligent systems improving user experience through the integration of contextual data. This brings the following advantages: we allow for managing of imperfect data and flexible queries, and we resemble what happens in the process of communication among people in query processing systems. Moreover, users can enjoy friendly interaction with fuzzy databases.

\section{Background}

In diverse software applications the content is heterogeneous. Users interact with service and product pro- 
viders, governmental organizations, friends and colleagues, as well as sensing and actuation devices. Such extensive information constitutes an unprecedented opportunity for users, but at the same time there is high risk to be overwhelmed. [10].

There are novel paradigms supported by the development of Information and Communication Technologies (ICT), such as the Internet of Things (IoT) in which the pervasive presence of a variety of things or objects (Radio -Frequency Identification or RFID tags, sensors, actuators, smart phones, among others) around us is recognized. These objects are able to interact with each other and cooperate with their neighbors to reach common goals [11].

Another emergent paradigm is the web squared or $\mathrm{Web}^{2}$ (web $2.0+$ world). This approach is aimed at integrating web and sensing technologies together so as to enrich content provided to users. This is obtained by taking into account information about the user context collected by the sensors (microphone, cameras, GPS, among others) deployed in the user terminals [11].

Additionally to IoT and $\mathrm{Web}^{2,}$ other approaches such as "Web of Sensors" or "Ambient Intelligence (AmI)" emphasize the trend towards a tighter connection between the cyberspace and the physical world. One of the reasons for this is an unprecedented explosion of mobile data volumes known as ambient data [12].

Thus, applications would be sensitive to user needs, capable of anticipating his/her behavior, requirements or preferences; interacting with people in a userfriendly way, and even capable of expressing, recognizing and responding to emotions [13].

These approaches recognize the contextual component of knowledge, which may be of use to extract and present chunks of knowledge, thus allowing for information filtering, focusing and reduction [10].

Consequently, user heterogeneity would imply that different users may find dissimilar things relevant when searching because of distinct preferences and goals. Particularly, when there is considerable amount of information available, it is important to provide the right answer to a specific user rather than the exact same answer to everyone based on the same query.

In the scientific community of database an issue of interest is the incorporation of user preferences in query answering to provide user customized results [14]. Furthermore, the authors in [15][16][17][18][19] have incorporated the context (in an explicit or inferred way) to establish user contextual preferences.

The integration of context-awareness to query processing is an issue addressed in ambient intelligence [20]. They intend managing the contextual data through a logic layer on top of the DBMS. An interesting paper showing the importance of the context into fuzzy querying is presented by Zadrozny et al [21]. They propose flexible queries through the use of contextual bipolar queries.

Thus, it is recognized that database applications require more intelligent information management to satisfy user demands in modern informatics systems. We consider important to integrate approaches and experience from diverse computational areas to address this issue. On next sections, we address the query rigidity problem in database systems through the integration of the context and management of fuzziness to develop intelligent, flexible and user customized systems.

\section{Theoretical Framework}

We propose to develop intelligent database systems with the support of Object Oriented Data Modeling, Fuzzy Databases, and the Computational Theory of Perceptions (CTP) [22].

We want to achieve context-aware queries affecting both the results and presentation of queries. To do this, we intend to manage imperfect and contextual data to benefit user information over the efficiency of the query.

Besides the traditional exact match paradigm in Database Systems, we suggest a different approach to obtain custom query answers affected by the user context that interacts with fuzzy databases: a Context-Aware Flexible Model. In next sub-sections we describe the theoretical bases of this model.

\subsection{Interaction Schema}

In figure 1, we illustrate a user general interaction schema with a proposed context-aware fuzzy database system. It can be noted that the system takes into account the:

- Traditional input (data or query)

- Explicit user context such as profile, role, state (physical, emotional or social), activity, situation or mobility

- Implicit context provided by the environment surrounding the user such as location, time, weather, application, device type, connection or web services

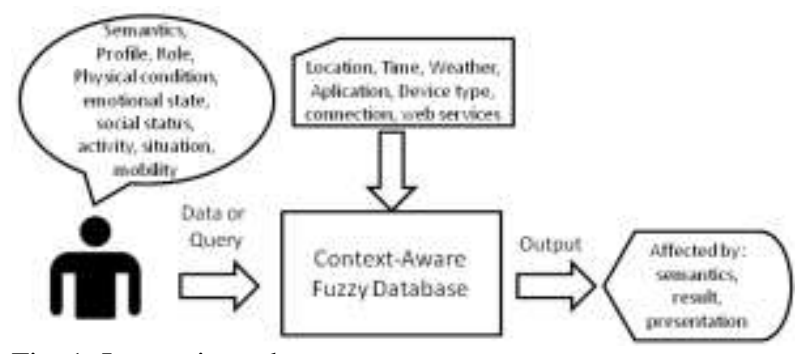

Fig. 1: Interaction scheme.

In these conditions, the system output can be affected in its semantics, results (size, precision, order, ranking or type); and presentation (output format or media type).

The designed model is customizable. That is, the system designer can make adjustments that she/he considers relevant according to the application domain. For example, the designer can remove the role or user profile without affecting the aim of the model.

We take hospitals as example of application domain. The broad features of hospitals relevant for the application of the proposed model are: the distributed nature of information, the need for collaboration, mobility (personnel and devices), and the need to access medical in- 
formation in a timely fashion for decision-making [13].

Additionally, we consider that the healthcare area is an example where the decisions are based on imperfect data and it is very important to take into account the perceptions of the diverse interested people.

\subsection{Context-Aware Flexible Model}

Once the fuzzy database system user general interaction schema is known, we explain the theoretical model to implement the context-aware fuzzy database system.

In Figure 3, we show the framework proposed by [23] to connect context and databases.

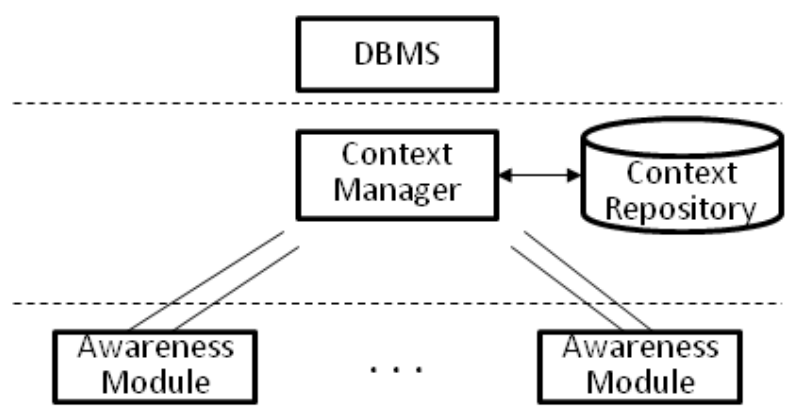

Fig. 2: Connecting Context and databases [23].

The components presented in the figure are the following:

- Awareness Modules communicate with data sources (such as sensors) and updates are propagated to the Context manager

- The Context Manager administers the context related information

- The Context Repository stores context data

There are two ways of integrating the context into a DBMS: 1) The Context Manager could be part of the DBMS, or 2) The Context Manager could be seen as a mediator in a middle layer.

In our proposal we choose the second way with the advantages provided by the use of an Object Relational DBMS (ORDBMS), those are:

- We employ Fuzzy Database in the DBMS layer, so we can store imperfect data and perform fuzzy queries (flexibility)

- We use standard sentences of SQL, trying to be as non-intrusive as possible in standard sentences used by users (transparency)

- We reduce impact on performance offering new facilities to users (low cost).

Thus, we design two Context Awareness Modules, they are: the Context Awareness Storage (CAS) module and the Context Awareness Retrieval (CAR) module; they both comply with the principles of the Computational Theory of Perceptions (CTP) and they constitute the Intelligent Modules that allow to input and to query data using linguistic labels associated to a fuzzy domain.

The CAS module allows to insert data in language that is close to user needs, the linguistic labels are transformed according to the user context, and then, raw data is stored as objects into the database (such as trapezoids). The CAR module lets retrieval of raw data and transforms them into linguistic labels. Then, the query results are displayed in the user defined language.

In our model we use the Context Awareness Support Manager (CASM) for managing the Contextual Catalog to infer behavior according to the context. The CASM constitutes the knowledge meta-base used by the CTP modules to act as Context Aware System intelligent agents.

In figure 3, we show the proposed theoretical model. The components of the model are described below.

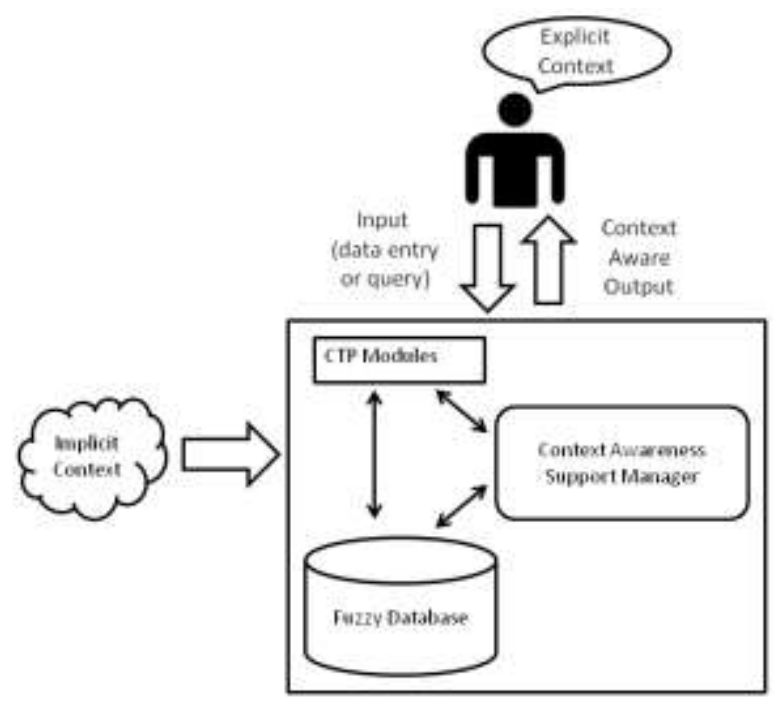

Fig. 3: Model for Context-Aware Fuzzy Database Applications.

- $\quad$ Context Awareness Support Manager (CASM): It is responsible for managing the Contextual Catalog, the user actions $\log$ and the rules to infer behavior according to the context. This module becomes the "Expert" that lets the CTP modules to make their transformation jobs

- Fuzzy Database: It manages imperfect data by means of the Computational Theory of Perceptions [20]. In this way, users could define new attribute types extending created fuzzy domains (categorical, discrete, continuous or conjunctive) and they could use different methods (defined for each fuzzy domain) to compare these attribute types

- Explicit Context: Contextual data provided by users such as semantics, role, state (physical, emotional or social), activity, situation or mobility

- Implicit Context: Contextual data provided by environmental sensors or software agents such as location, time, weather, application, and device or connection type

Additionally, we define a Context-Aware Application Module where users set specific features of the application domain. For example, each user could define proper semantics for a determined attribute. Additionally, we consider that an expert or administrator could define default semantics.

With this model, we achieve that a query submitted to the same database produces different outputs according to the context in which that query is performed. 


\section{Context-Aware Fuzzy Database web Application}

In this section we describe the use of the theoretical model applied in a specific domain, which is the Children's Orthopedic Hospital, located in Caracas, Venezuela. The hospital mission is to care for poor children with musculoskeletal problems. Over the years, it has adapted to the needs of the population expanding the range of its services, to even serving adults.

The hospital has a gait analysis laboratory which is used to diagnose diseases of the musculoskeletal and neuromuscular system The analysis performed in this laboratory allows for an accurate patient diagnosis to those with walking problems, allowing for the planning of surgery and orthopedic indications with scientific support. We focus on the Joint Physical Examination carried out as part of a more integral test that includes signal reports, $\mathrm{x}$-rays, and patient's gait videos

We propose a Context-Aware Fuzzy Database web application with mobile interface to managing JPE information. Nowadays, this information is managed through an application using the Microsoft ${ }^{\circledR}$ Access Database. The data are collected using two application forms called: "Patient Identification" (personal data, background and references), and "Joint Physical Examination" (JPE) where the biomechanical evaluation characteristics observed in the patients joints are stored.

A physical therapist (or physiotherapist) assesses the biomechanical characteristics in the joints of the lower limbs (hip, knee, ankle and foot) of the patient and the characteristic motion associated with each one. For example, the hip, an enarthrosis, a type of spheroidal (or ball and socket) joint, that is because it can perform all joint movements such as flexion, extension, abduction, adduction, internal rotation, and external rotation. Then, the physical therapist assesses joint range, selectivity/strength and muscle tone.

We analyze 143 attributes of JPE and we categorize them by fuzzy domains [9] or traditional (crisp) domain. There are attributes such as weight and height that are fuzzy because they are exactly measured in origin but may be fuzzy on queries. That is, we allow to define linguistic labels for these attributes using a continuous fuzzy domain and then use these labels in fuzzy queries. In Table 1 we present some of the attributes and their fuzzy domains.

We use continuous, categorical, discrete and conjunctive fuzzy domains. Moreover, there are case where value attributes are crisp but fuzzy on queries (for example height and weight). The managing of these fuzzy domains in the backend is defined in [9].

A Medical Interpreter uses this data along with patient background and other studies for the diagnosis and makes decisions about treatments. Through the use of CTP, the system can manage different linguistic labels according to user preferences semantics (user context), extending the results that traditional systems provide, and achieving a better understanding of the mechanisms of human reasoning.

We implement other context awareness characteristics of the JPE web application. We will explain these in the next section.

\begin{tabular}{|l|l|l|}
\hline Attribute & Joint & Fuzzy Domain \\
\hline Flexion & Knee & Continuous \\
Flexor/Extensor tone & Knee & Discrete \\
Abduction/Adduction & Hip & Continuous \\
Flexion/Extension Tone & Hip & Discrete \\
Dorsiflexion (Dor) & Ankle & Continuous \\
Plantar flexion (PF) & Ankle & Continuous \\
Inverters/Evertors Tone & Ankle & Discrete \\
Rearfoot/Forefoot & Foot & Continuous \\
Break Reducible & Foot & Continuous \\
Gait type & & Categorical \\
Mobility Aids & & Conjunctive \\
\hline
\end{tabular}

Table 1: Attributes of JPE and its fuzzy domains.

\subsection{Description of the Application}

The components of the JPE application and its relationship with the Context-Aware Fuzzy Database are showed in figure 4 . The context awareness characteristics of the JPE application are described next.

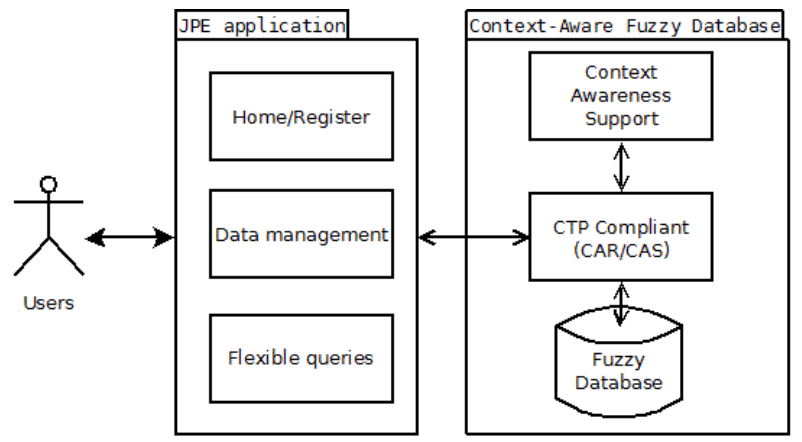

Fig. 4: JPE application and Context-Aware Fuzzy Database.

- Role: As a physiotherapist the user can enter all the data related to a profile. Additionally, she/he can enter patient and JPE data, and run queries (traditional or fuzzy). As a medical interpreter the user can enter only profile data and run queries. As an administrator the user sets configuration parameters for the application such as default semantics.

- Profile: Each user can define trapezoidal membership functions and corresponding linguistic labels (continuous fuzzy domain), resemblance matrix (categorical fuzzy domain) and/or description matrix (discrete fuzzy domain). Then, each user obtains personalized answers according to her/his semantics.

- Device type: The JPE System recognizes when a user connects with an Android OS mobile device. Then, the mobile interface (which is responsive to the screen size) is activated.

- Size: So, if a user uses a mobile device to browse the query answers, then our application displays maximum of eight rows. In case of a fuzzy query with only a membership degree, we 
use the threshold to show the more interesting answers to the user.

- Contextual dimensions: A user administrative interface allows each user (with a role of administrator) to set parameters for the application; that is, she/he can set default semantics and/or contextual dimensions (like time, location, state or task), fuzzy domains, and contextual dependencies.

First, the system identifies the device type and then it uses the corresponding application interface (desktop or mobile). When the user registers he/she has to assume a role: physiotherapist or medical interpreter. In figure 5 we show an example of the application interface for desktop devices.

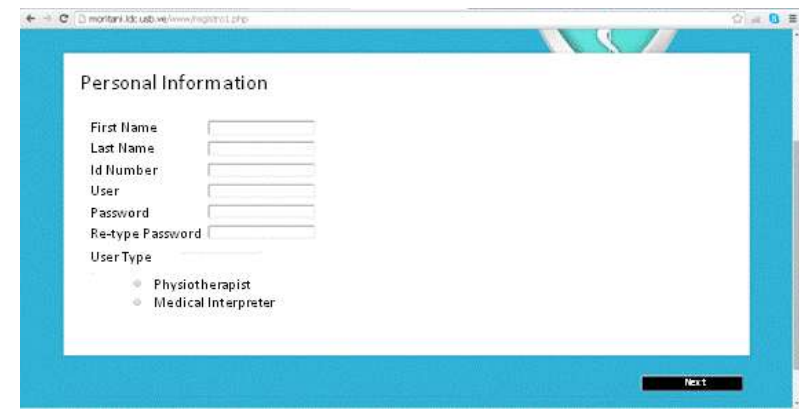

Fig. 5: Example of interface for JPE web application.

In figure 6 , we show the elements of the mobile application interface. The six icons at the top correspond to home (profile), adding patient, new Joint Physical Examination, queries, delete patient and quit. The output displayed corresponds to a query about the JPEs made by the physiotherapist "Ruben Serradas". It is important to note that because of the number of attributes of the JPE, most of the data are entered using the desktop application interface.

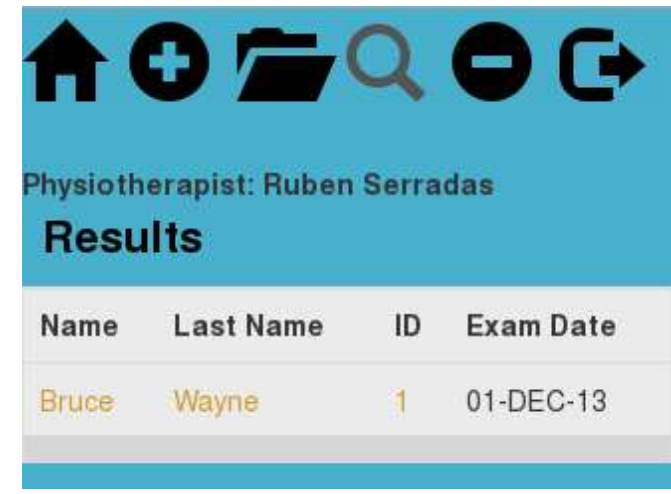

Fig. 6: Application interface for mobile devices.

Figure 7 shows answer to the query: Thin patients with normal gait, where thin and normal are linguistic labels defined by physiotherapist Ruben Serradas. It is important to note that weight is an fuzzy attribute on queries and gait type is a categorical fuzzy domain.

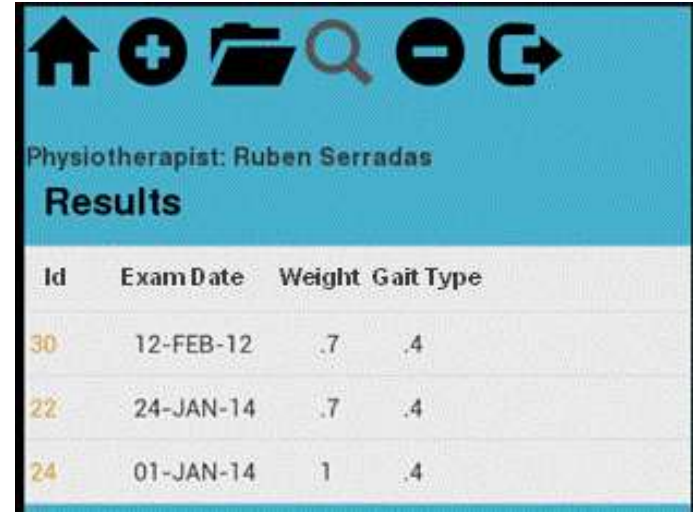

Fig. 7: Answers for query: thin patients with normal gait.

In Figure 8, we exemplify the results to the same query above but the linguistic labels were defined by Doctor Bennet.

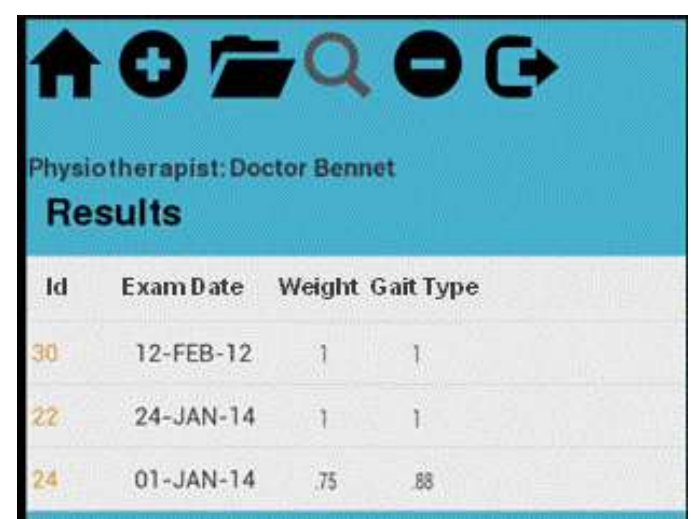

Fig. 8: Answers for Doctor Bennet's query.

Another type of query is the comparison among patients who use mobility aids. In this case, the system asks for patient identification and then, makes the comparison of conjunctive fuzzy domain with the others patients into the database. For mobile devices, the system, as part of context-awareness, shows at most eight rows because of the limitation of the display. For this aim the system establishes a threshold and displays the result in descending order of membership degree. In Fig. 9 we show the results for patient Maria Pérez.

Another example is when a user runs a query but in different contexts. In Fig. 10 the user has normal linguistic label for Hip flexion in general examination, and in Fig. 11 the user has another normal linguistic label for Hip flexion in post surgical examination. Then the results obtained for the Query "Patients with normal Hip flexion" are different despite the having the same raw data.

In previous work [9], we show examples using the SQL:99 standard of how the user can define semantics of linguistic labels with trapezoidal membership functions. In the present work the user interacts with the system through user-friendly applications where the use of SQL:99 is transparent for her/him. 


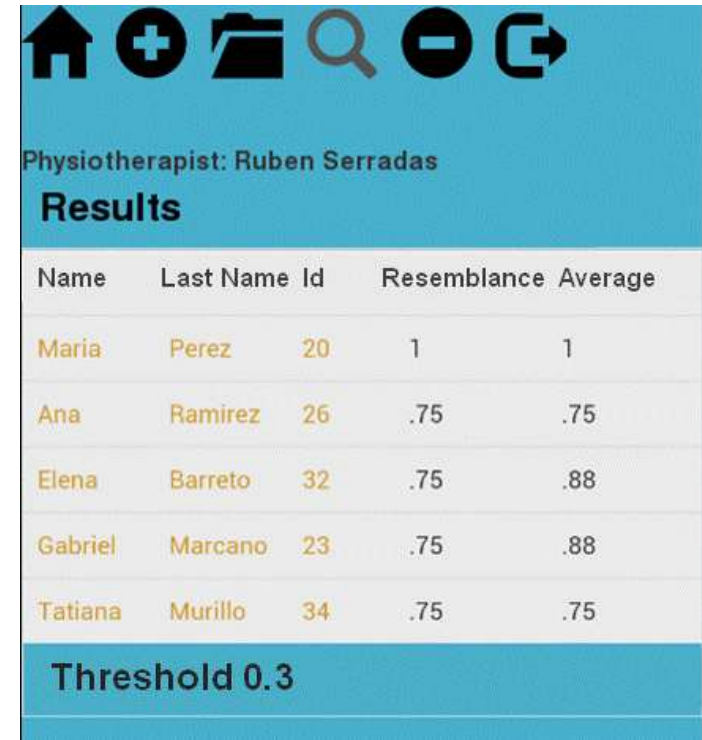
aids).

Fig. 9: Resemblance with Maria Perez patient (mobility

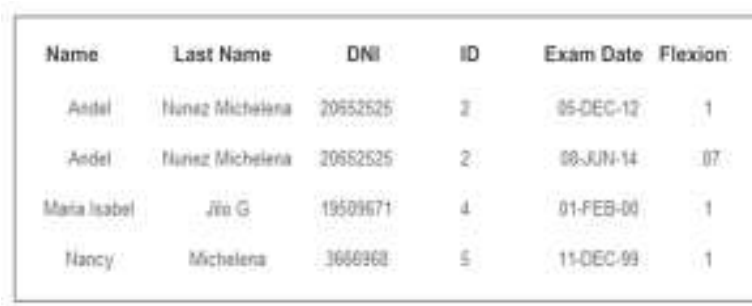

Fig. 10. Patients with Normal Hip flexion (general examination)

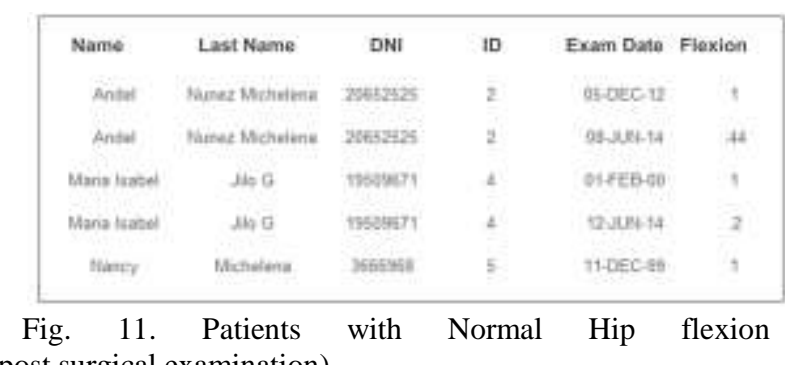

(post surgical examination)

When a different user, who has different definitions of each linguistic label, connects to the system and makes a query, the answers are showed according to the semantics of the last user. In this way, the connected user affects the semantics of linguistic labels and the answers of the query are context-aware.

In general, each user could define the semantics of linguistic labels for continuous fuzzy domains according to his/her needs. In addition, we consider that an administrator user (expert or system designer) would make the definitions that the system will use if the user does not make them.

\section{Conclusions and Future works}

We present a Context-Aware Flexible Model to address the problem of rigidity produced by the exact match paradigm in traditional Database Systems. The main contributions of our proposal are summarized in:

- Managing of multiple contextual dimensions
- Definition of context dependencies.

- Managing of imperfect data using fuzzy domains.

- The personalization of semantics of linguistic labels used, extending the results that traditional systems provide, and achieving a better understanding of the mechanisms of human reasoning.

We aim at developing intelligent systems improving user experience through the integration of contextual data. This brings the following advantages: users can enjoy friendly interaction with fuzzy databases, we allow for managing of imperfect data and flexible queries, we give significance to the usefulness for the query result user, we resemble what happens in the process of communication among people in query processing systems.

We develop a friendly web application to allow the interaction of non-technical users with the system. The application was developed for the Venezuelan Children's Orthopedic Hospital focused on the Joint Physical Examination (JPE) accomplished in the gait Analysis Laboratory.

This application shows the convenience and feasibility of the proposed Context-Aware Flexible Model. The context awareness characteristics of the JPE application take into account the contextual dimensions: user role, user profile, device type, size of results and any other that an administrator could define. Particularly, users can define semantics of linguistic labels, allowing custom input data and getting flexible queries according to user needs.

For future work we intend to use sensor data for mobile applications. Another issue is to infer contextual data using the data provided by Context Awareness Support Manager, such as user log actions. Additionally, the application of the model using the non-SQL paradigm could be explored, that is because of the large amount of data provided by sensors and social networks in the era of big data.

\section{Acknowledgement}

This work has been partially supported by the Spanish Government under the project TIN2014-58227 "Descripción lingüística de información visual mediante técnicas de minería de datos y computación flexible". We would like to thank Fundación Carolina for their support to develop this research.

\section{References}

[1] G. Koutrika, Personalized DBMS: an Elephant in Disguise or a Chameleon?, IEEE Data Eng. Bull. 34.2: 27-34, 2011.

[2] F. E. Petry, Fuzzy Set Theory Utility for Database and Information Systems. In On Fuzziness: 547552, Springer Berlin Heidelberg, 2013.

[3] Z. Ma and L. Yan, editors. Advances in Probabilistic Databases for Uncertain Information Management. Series: Studies in Fuzziness and Soft 
Computing, Vol. 304. Springer Publishing Company, 2013.

[4] M. Koyuncu, Fuzzy Querying in Intelligent Information Systems. In T. Andreasen et al. (Eds.), proceedings of the $8^{\text {th }}$ international conference on flexible query answering systems (FQAS 2009), Lecture Notes in Artificial Intelligence 5822, pages 536-547, Springer-Verlag, 2009.

[5] J. Cadenas, N. Marín and M. Vila, Fuzzy domains with adaptable semantics in an object-relational DBMS. In H. Christiansen et al. (Eds.): Flexible Query Answering Systems (FQAS); Lecture Notes in Computer Science 7022, pages 497- 508, Springer Berlin Heidelberg, 2011.

[6] F. Berzal, N. Marín, O. Pons, M.A. Vila. Managing fuzziness on conventional object-oriented platforms. International Journal Intelligent Systems; Vol. 22, pages 781-803, 2007.

[7] C.B. Almazan, Rover: architectural support for exposing and using context. Doctoral Thesis. Universidad de Maryland, 2010.

[8] M. A. Muñoz, M. Rodríguez, J. Favela, A.I. Martinez-Garcia, and V.M. Gonzalez, Context-aware mobile communication in hospitals. IEEE Computer Society, 36(9): 38-46, 2003.

[9] J.T. Cadenas, N. Marín and M.A. Vila, ContextAware Fuzzy Databases. Applied Soft Computing, 25:215-233, Elsevier, 2014.

[10] G. Orsi, and L. Tanca, Context Modelling and Context-Aware Querying (Can Datalog Be of Help?). O. de Moor et al. (Eds.): First International Workshop Datalog, Lecture Notes in Computer Science 6702, pages 225-244, SpringerVerlag, 2011.

[11] L. Atzori, A. Iera and G. Morabito, The Internet of Things: A survey, Computer Networks, Volume 54, Issue 15, pages 2787-2805, ISSN 1389-1286, 10.1016/j.comnet.2010.05.010, 2010.

[12] KT. Huynh, B. Finance and M. Bouzeghoub, Towards an ambient data mediation system, 2nd International Workshop on Information Management for Mobile Applications (IMMoA'2012), 2012.

[13] F. Sadri, Ambient intelligence: A survey. ACM Computing Surveys (CSUR), 43(4):36, 2011

[14] K. Stefanidis, G. Koutrika, and E. Pitoura, A survey on representation, composition and application of preferences in database systems. ACM
Transactions on Database Systems (TODS), vol. 36(3):19, 2011.

[15] A. Miele, E. Quintarelli, E. Rabosio, L. and Tanca, A data-mining approach to preference-based data ranking founded on contextual information. Information Systems, 38: 524-544, Elsevier, 2013.

[16] A. Hadjali, A. Mokhtari, and O. Pivert, A FuzzyRule-Based Model for Handling Contextual Preference Queries, International Journal of Computational Intelligence Systems, 5(4): 775-788, 2012.

[17] P. Roocks, M. Endres, S. Mandl, W. and Kießling, Composition and Efficient Evaluation of ContextAware Preference Queries. Proceedings of the 17th Int. Conference DASFAA, Busan, South Korea, pages 81-95, Springer, 2012.

[18] F. Wenzel, M. Soutschek and W. Kießling, A Preference SQL Approach to Improve ContextAdaptive Location-Based Services for Outdoor Activities. G. Gartner, F. Ortag (Eds.), Advances in Location-Based Services, Lecture Notes in Geoinformation and Cartography, pages 191-207, Springer-Verlag Berlin Heidelberg, 2012.

[19] J. Levandoski, M. Khalefa and M. Mokbel, An Overview of the CareDB Context and PreferenceAware Database System. Proceedings of the Very Large Databases (VLDB), 3(2):1529-1532, 2010.

[20] A. Bunningen, L. Feng, P. Apers. A context-aware preference model for database querying in an ambient intelligent environment. In: S. Bressan, J. Kung, R. Wagner (Eds.). Database and Expert Systems Applications, Lecture Notes in Computer Science. Springer Berlin Heidelberg., vol. 4080, pages 33-43, 2006.

[21] S. Zadrozny, J. Kacprzyk, M. Dziedzic, M. \& G. De Tré. Contextual bipolar queries. In M. Jamshidi, V. Kreinovich, \& J. Kacprzyk (Eds.), Advance Trends in Soft Computing: Proceedings of WCSC 2013, December 16-18, San Antonio, Texas, USA. Springer, Vol. 312, pages 421-428, 2014.

[22] L. Zadeh, A new direction in AI: Toward a computational theory of perceptions. AI Magazine 2001;22(1):73-84, 2001.

[23] E. Pitoura, K. Stefanidis, and A.B. Zaslavsky, Context in Databases, Technical Report TR 200419, Department of Computer Science, University of Ioannina, Greece, 2004. 